\title{
Post-resectional pelvic recurrences of rectal
}

\section{cancer}

\author{
Adnan Al ALOUL ${ }^{1,2}$, Dan Florin UNGUREANU ${ }^{3}$, Nicolae BACALBASA ${ }^{4,5}$ \\ ${ }^{1}$ Department of Surgery, Ramnicu Sarat County Hospital, Buzau, Romania \\ 2Doctoral School, "Titu Maiorescu" University, Bucharest, Romania \\ ${ }^{3}$ Department of Surgery, "Titu Maiorescu" University, Bucharest, Romania \\ ${ }^{4}$ Department of Obstetrics and Gynecology, "Carol Davila" University of Medicine and Pharmacy, \\ Bucharest, Romania \\ ${ }^{5}$ Department of Visceral Surgery, Center of Excellence in Translational Medicine Fundeni Clinical \\ Institute, Bucharest, Romania
}

\begin{abstract}
- ABStract
Introduction. Pelvic recurrence is not a rare event after resection with curative intent for rectal cancer originating from different segments of the rectum (lower, intermediary and superior part).

Material and methods. This retrospective observational study included 106 patients; among these cases there were 79 patients who accepted surgical treatment of rectal cancer (treated in a governmental hospital between 2014 and 2017) and who were submitted to anterior resection with Hartmann's procedure $(6.5 \%$ of patients), anterior resection of rectosigmoid with colorectal anastomosis ( $78 \%$ of cases) and abdominoperineal resection ( $15 \%$ of cases).

Results. After a 2 year follow-up, pelvic recurrence was reported in 11patients 14\% of cases: $33 \%$ rate of recurrence after Hartmann procedure, 9\% rate of recurrence after abdominoperineal resection, and $10 \%$ rate of recurrence after anterior resection of rectosigmoid with colorectal anastomosis. 39 patients ( $49 \%$ of cases) had been submitted to preoperative radiotherapy: the pelvic recurrence rate among these cases was of $11 \%$ (9 patients). The rate of recurrence ( $R R$ ) was also significantly influenced by the stage at diagnostic: stage III had $R R=52 \%$ of cases, stage II had $R R=41 \%$ of cases and stage I had $R R=0 \%$ of cases). The survival rate among surgically treated patients after 1 one year was $86 \%$, and $80 \%$ in the first 2 years after treatment.

Conclusions. Rectal cancer diagnosed in advanced stages has a high recurrence rate. A low recurrence rate indicates successful curative surgical treatment. The highest recurrence rate was reported after Hartmann procedure (which was usually performed as an emergency operation for locally advanced lesions).
\end{abstract}

Keywords: rectal cancer, tumor resection, recurrence rate

\section{INTRODUCTION}

Rectal cancer is an important cause of morbidity and mortality worldwide, given the late stage diagnosis (due to the lack of symptoms). The rate of pelvic recurrence is significantly influenced by the stage of disease and by the type of treatment; therefore cases diag- nosed with locally advanced lesions submitted to per primam surgical therapies report a higher risk of recurrence (1).

According to the macroscopic aspect, colon cancers can be divided into 4 types: ulcerative form: crateriform tumors with budded edges, having the typical appearance of a malignant ulceration; shape characteris- 
tics: raised and irregular edges (defining circular / oval shapes), often with necrotized base; the lesion has a tendency to deeply infiltrate the colon; the ulcerative form has a higher risk of perforation when compared to other forms; stenotic / annular shape: circular lesion, varying extent, tendency to ulceration; the extended form is more common in the rectum and the short forms are more common in the transverse and descending colon; polypoid shape: proliferative lesion, protruding in the intestinal lumen; lobulated appearance with variability in lobe size; possible ulcerations on the surface; more frequent manifestation in the cecum and the ascending colon and diffuse infiltrative form: a form of rectal cancer which extents on the length of the intestinal wall with at least 5-8 cm, rarely ulcerative areas; the rarest form of all the four forms $(2,3)$.

Generally speaking, colon tumors are infected, their volume being amplified by inflammatory peritumoral sclerolipomatosis: the infection is responsible for the retraction of the tumor-bearing segment, thus resulting in its reduction of caliber and length (2).

When it comes to the ways of dissemination, multiple routes have been incriminated: hematogenous, lymphatic and peritoneal route as well as local extension. Dissemination by hematogenous route occurs by venous propagation of the tumor thrombi; this way of propagation predominantly produces liver and lung metastasis (through the veins of the Retzius retroperitoneal system and through the lumbar veins); once the liver and lung are affected, tumoral cells metastasizes to any other viscera (especially at the level of well vascularized organs). Dissemination by lymphatic route consists of initial development of malignant cells at the level of the lymph nodes from the colon wall and in the epiploic lymph nodes (with successive or discontinuous/skipped metastasis pattern); the walls of the lymphatic vessels are also affected by lymphatic tumor permeation resulting in neoplastic lymphangitis. Dissemination by local extension occurs directly (by proliferation from close to closer, with a step-by-step invasion of colonic parietal structures and then of neighboring structures and organs while dissemination in the peritoneal cavity occurs by tumor exfoliation and intraperitoneal grafting leading to the apparition of peritoneal and epiploic metastases $(2,3)$.

The clinical manifestations of colon cancer are either absent (for a long time) or are nonspecific and easily neglected or superficially diagnosed and treated. The most commonly reported symptoms are represented by: pain (the site of pain is located either above the site of the tumor or near it, transit disorders (with onset usually without any apparent cause; the mainly form of transit disorder is an alternation between constipation and diarrhea); blood loss (which usually man- ifests as occult / microscopic bleeding - rarely observed by the patient or as rectal bleeding when the tumor is low (4).

As for the clinical examination, there are two aspects to which the clinician should pay attention: to palpate the last 7-8 $\mathrm{cm}$ of the rectum from the anus and, in women, to examine the vagina (which can offer information about the tumor, by palpation) (4).

When it comes to the standard, international classifications, the most commonly used systems are represented by the TNM and AJCC classifications $(5,6)$.

As for the most efficient therapeutic strategies, surgery alone or in association with radiation therapy represent the safest procedures; when it comes to the surgical treatment, one of the most important features which influence the long term outcomes is represented by the radicality of resection; therefore, it is strongly to resect the entire mesorectum, with safety margins of at least $8-10 \mathrm{~cm}$ proximally and at least $2 \mathrm{~cm}$ distally. Meanwhile, the type of surgery (anterior resection of with Hartmann's procedure, anterior resection of rectosigmoid with primary colorectal anastomosis, abdominoperineal resection) also seems to influence the outcomes (7-10).

The objectives of the current study are to investigate the most commonly performed types of surgical procedures for rectosigmoidian cancer and to analyze the risk factors associated with a higher rate of postoperative risk of local relapse.

\section{MATERIAL AND METHODS}

We conducted a retrospective observational study on 79 patients with surgically treated rectal cancer at "Angelescu" Hospital (Bucharest, Romania) and University Emergency Hospital (Bucharest, Romania), between 2014 and 2017 by using these three techniques: anterior resection with Hartmann procedure, anterior resection with primary colorectal anastomosis and abdominoperineal resection (7).

The medical data was extracted from the archives of medical files (from the previously listed hospitals) of the patients diagnosed with rectal cancer (in the previously mentioned time interval). We've selected the medical files of 79 patients (with dynamic follow-up), from which the parameters used for this study were extracted. General data such as age, sex, living environment, physical activity, family history, personal pathological antecedents, behaviors (smoking, alcohol, drugs) as well as specific data - preoperative cancer stage (at the date of the initial cancer diagnosis), location of the neoplasm, presence of positive pelvic lymph nodes, presence of peritoneal carcinomatosis, invasion of the surrounding organs, associated comorbidities, type of surgical technique or type of procedure (elec- 
tive versus emergency surgery). Meanwhile data extracted from the postoperative follow up controls (such as clinical, imagistic studies), association of radiotherapy or recurrence diagnosis were also retrieved.

\section{RESULTS}

The study initially included 106 patients, among these cases there were 79 patients submitted to surgery for rectal or rectosigmoidian cancer; among surgically treated patients we identified 11 cases who developed recurrent disease. Meanwhile, at the end of the study 16 deaths of disease were reported. Among the 79 patients included in the current study sex ratio male to female was of $57 \%$ to $43 \%$.

The variation of incidence with age group on the total of 106 patients demonstrated that this diagnosis was most commonly reported in the 70-79 age group, followed by the 60-69 age group. The 70-79 age group represented $33.5 \%$ of cases, the $60-69$ decade represented $28 \%$ of cases, the 50-59 decade represented $23.5 \%$ of cases, the $40-49$ age group represented $4.5 \%$ of cases while $1,5 \%$ of patients were older than 80 years. The distribution according to the age subgroup is presented in Figure 1.

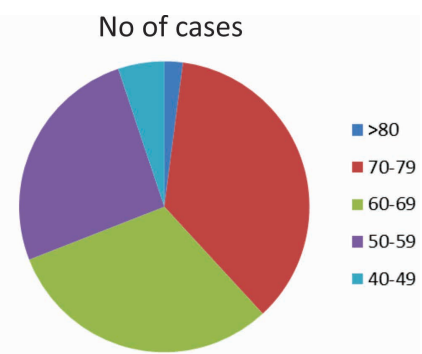

FIGURE 1. Patients' distribution according to the age group

The distribution of patients according to their living environment: there was an urban-to-rural ratio of 3-to-1. This ratio may be explained by the diet (which is healthier in rural areas), habits (which are also healthier in rural areas) and exposure to carcinogens (which is higher in the urban areas).

From the total of 106 patients, the distribution of cancer stages was the following: $23 \%$ of cases were diagnosed in stage I, $48.5 \%$ of patients were diagnosed in stage II, $15.5 \%$ of cases were diagnosed in stage III and $14 \%$ of patients were diagnosed in stage IV. The distribution according to the stage at diagnostic is illustrated in figure 2.

When it comes to the clinical manifestations, $62 \%$ cases had abdominal pain, $69 \%$ of them had rectal reactions, $51 \%$ of patients had transit disorders and $40.5 \%$ of patients had rectal tenesmus (this latter symptom was reported more frequently in rectal tumors with low positions). All patients underwent abdom-

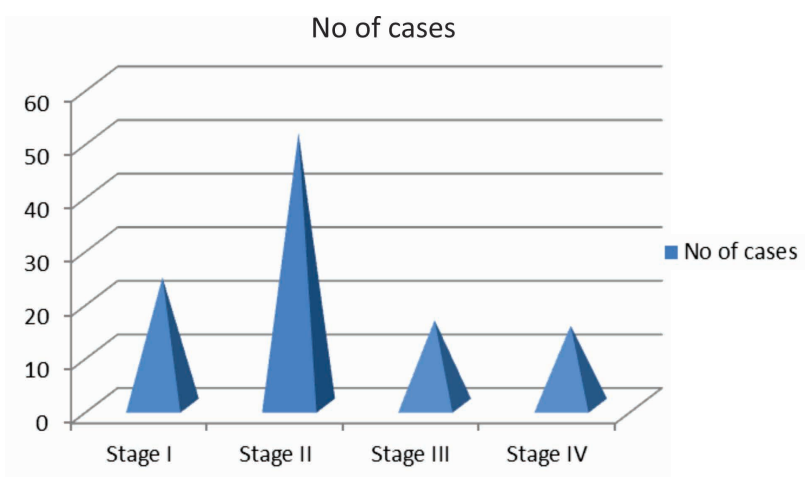

FIGURE 2. Patients' distribution according to the stage at diagnostic

inopelvic ultrasound, upper-abdominal and pelvic computed tomography (CT); chest CT was performed in only $65.6 \%$ (this investigation was very useful in detecting distant metastases and, in many cases, lymphadenopathies were detected, but no distinction could be made between inflammatory or malignant lymphadenopathies); total colonoscopy or sigmoidoscopy were performed in $77 \%$ of patients ( $7 \%$ of these procedures could not be completed because of intestinal obstruction which imposed emergency surgery).

14 patients were initially diagnosed with stage-IV rectal cancer and therefore were not submitted to surgery (these 14 patients had metastases in various organs: in the genitals or other surrounding organs in seven patients, hepatic metastases in five patients, peritoneal carcinomatosis in three patients and both liver and lung metastases in two 2 patients.

According to the location of the tumor, the 79 cases were classified in three categories: cases presenting lower rectal lesions - located at up to $5 \mathrm{~cm}$ from the anal orifice ( 30 patients $=38 \%$ of cases), cases presenting middle rectal lesions - located between 5 and $10 \mathrm{~cm}$ from the anal orifice ( 12 patients $=15 \%$ of cases) and cases presenting lesions at the level of the upper rectum at $10-15 \mathrm{~cm}$ from the anal orifice $(37$ patients $=$ $47 \%$ of cases)

Seven cases had tumors localized at the level of the rectosigmoid junction (which was also classified as upper rectum cancer); from the analysis of the data referring to the tumor site, stage and clinical symptoms, certain conclusions can be drawn: a large number of cases -51 patients $=48 \%$ of cases) were diagnosed with stage II lesions located at the level of the upper rectum due to bleeding; 15 cases were diagnosed with stage-IV rectal cancer (in all the anatomical regions of rectum); most cases were admitted with abdominal pain, bleeding and transit disorders. When it comes to the type of surgical procedure, it was established depending on the tumor stage, tumor site and the level of emergency (when intestinal occlusion occurred).

In the group of patients submitted to surgery, there were 12 cases which underwent abdominoperineal re- 
section (15\% of cases), 62 cases which underwent anterior rectosigmoidian resection with colorectal or coloanal anastomosis (78\% of cases) while the remaining cases were submitted to Hartmann procedure. Among the 62 cases submitted to resection followed by colorectal anastomosis diverting ileostomy was needed in 12 cases; the decision of performing of an ostomy was taken in cases in which poor vascularization, malnutrition or poor preoperative preparation was observed and was followed by reintegration at six to eight weeks after resection. Hartmann rectosigmoidian resection was the procedure of choice in cases presenting stage-IV rectal cancer. The illustration of distribution depending to the type of procedure is represented in figure 3 .

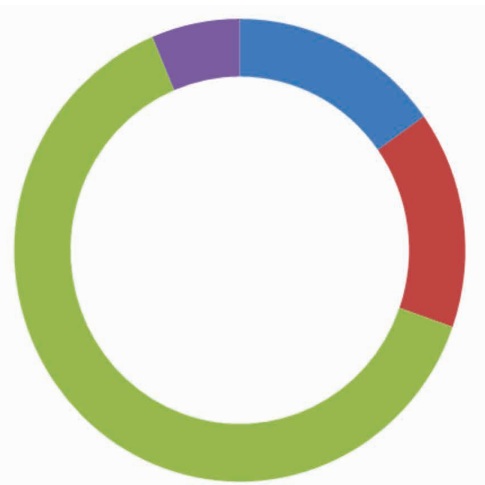

abdominoperineal resection

- Rectosigmoidian resection with anastomosis and diverting ileostomy

Rectosigmoidian resection with anastomosis without diverting ileostomy

- Hartman procedure

FIGURE 3. Patients' distribution according to the type of surgical procedure

Patients who underwent surgery were followed for at least 2 years (by regular check-ups) in order to observe the apparition of local or distant recurrences. In the 79 patients (which underwent surgical resection of rectal cancer), total relapses occurred in $13 \%$ of cases; this percentage is better than the ones reported in other studies in which the recurrence rate ranged between 14 and 20\%. Patients underwent postoperative follow up (at 1 month, 3 months, 6 months, 1 year and 2 years), consisting of anamnesis, clinical examination, colonoscopy; abdominal and pelvic ultrasound; chest radiography; abdomen, pelvis and thorax $\mathrm{CT}$, urography and whenever a diagnostic doubt was identified, cystoscopy.

$49 \%$ of patients received preoperative radiotherapy and $20 \%$ of them received postoperative radiotherapy: additionally, $38 \%$ of them also received chemotherapy and 10 patients $(12.7 \%)$ received a mixed oncological treatment (chemotherapy and radiation therapy). 24\% of the surgically treated patients who underwent pre / postoperative radiotherapy / chemotherapy had postoperative recurrences (distant metastases, local recurrences or both).

In our study rectosigmoid resection with colorectal anastomosis was associated with a $10 \%$ rate of local re- lapse/recurrence, abdominoperineal resection was associated with a local recurrence rate of $9 \%$ of all cases while Hartmann procedure was associated with a recurrence rate of $30 \%$.when it comes to cancer related death, In the $1^{\text {st }}$ year we've lost a total of 11 patients (two patients died immediately after surgery and other nine patients died within the first year) which indicates a survival rate (at 1 year) of $86 \%$ and a mortality rate of $14 \%$. Other five patients died within the $2^{\text {nd }}$ year after surgery resulting a survival rate (at 2 years) of $80 \%$.

\section{DISCUSSIONS}

The recommended check-ups after colorectal surgery are scheduled at 3, 6, 12 and 24 months and included the following steps: screening for abdominal pain, rectal bleeding, transit disorders, decreased appetite and weight loss; medical imaging: abdominal and pelvic ultrasound, endoscopic ultrasound, abdominal and pelvic CT, MRI (because it has a much higher sensitivity for pathological lymph nodes than other investigations). The American Society of Clinical Oncology (ASCO) has imposed clear steps for monitoring surgically treated patients so that to detect as early as possible any local recurrences and/or distant metastases: ASCO recommends abdominal, pelvic and thoracic CT at 6 months postoperatively, then annually for up to 3 years in patients with high-risk of cancer recurrence; ASCO recommends pelvic CT in all patients, especially in patients with elements of unfavorable prognostic. In our study, abdominal, pelvic and chest CT was performed in all patients for perioperative staging; abdominopelvic ultrasound was performed in all patients perioperatively; complete imaging (CT and ultrasound) was performed postoperatively at $53 \%$ of the surgically treated patients (as a criteria of ASCO) (8).

When it comes, to recurrent disease, this entity is defined as a local or regional relapse of a primary cancer (local or regional pelvic recurrence in the case of rectal cancer). Rectal cancer can also generate distant metastases that are considered general recurrence. In the current study local recurrence is defined as a recurrence after surgery (by using the three types of procedures mentioned above) and it is considered to be a recurrence of the primary tumor (which was initially removed) found at the level of the site of resection $(11,12)$, invading or not the surrounding organs (such as bladder, small intestine, retroperitoneal lymph nodes, remaining colon, prostate in men, uterus and vagina in women).

As for the role of complementary oncological treatment, several international studies have shown that pre- and post-operative radiotherapy and chemotherapy) bring great benefits for rectal cancer patients. There have been multiple randomized trials in recent years which demonstrated that preoperative radiation 
therapy may reduce local recurrences of rectal cancer from $27 \%$ to $11 \%$ with evidence of improving long-term survival rate from $48 \%$ to $58 \%,(8,13-17)$.

In our study, the percentage of recurrence-free patients reached a maximum of $95 \%$ in those patients who received preoperative radiotherapy (with only $5 \%$ of those patients having pelvic recurrences).

The rate of recurrence seems to be also significantly influenced by the initial stage at diagnosis. It is wellknown that the rate of local relapses (of rectal cancer) strongly depends on the depth at which the tumor penetrates the intestinal walls. In our study, 10 patients with stage II rectal tumor relapsed ( $41 \%$ of all relapsed patients); meanwhile 12 patients with stage III rectal tumor also relapsed ( $52 \%$ of all relapsed patients). No patient with stage-I cancer had relapsed in our study (this was expected because all studies from the literature show a recurrence rate below $0.5 \%$ in patients with stage-I rectal cancer). These data clearly show that the initial stage of the rectal cancer strongly influences the rate of local recurrence, with stage III having the highest recurrence rate and stage I having an almost zero recurrence rate $(15,16)$.
When it comes to cancer related mortality, it should not be omitted the fact that colorectal cancer is the second leading cause of death in patients with cancer, after lung cancer. Our results are comparable to a similar study in Britain (2005) where the survival rate was of $80 \%$ at 2 years (9).

\section{CONCLUSIONS}

Rectal cancer patients often reach a hospital in advanced stages of disease and are usually submitted to anterior resection; this procedure is also associated with the lowest recurrence rate (thus being best surgical method up to date); the preoperative radiotherapy significantly decreases the recurrence rate; the late cancer stage at initial diagnosis is the major risk factor for rectal cancer relapse; Hartmann procedure (which was performed as a matter of urgency, in old age groups) had generated the highest rate of rectal cancer recurrence.

Conflict of interest: none declared Financial support: none declared

\section{REFERENCES}

1. Dragomir C, Scripcariu V, Dajbog E. Cancerul de rect. Tratat de chirurgie generală. vol. IX, partea a II-a. Bucureşti: Editura Academiei Române, 2009:269-282.

2. Copotoiu $\mathrm{C}$. Tumorile rectale benigne şi maligne - Tratat de patologie chirugicalã (sub redactia N. Angelescu). Bucureşti: Editura Medicală, 2001.

3. Tinca A, Ion D. Cancerul rectal abordul multimodal actual. Bucureşti: Editura Universitară Carol Davila, 2019:57-63.

4. Vasile I, Munteanu M, Pasalega M, Gugila I. Semiologie chirurgicală, Craiova: Editura Medicală Universitară Craiova, 2005:230-242.

5. Schwartz SI. Schwartz's Principles of surgery, tenth edition, McGraw-Hill Education, 2015:1206-1214

6. American Joint Committee on Cancer, Seventh Edition. Springer, 2010:143-170. Available at: http://cancerstaging.org/ references-tools/deskreferences/Documents/ AJCC $\% 207$ th $\% 20$ Ed $\% 20$ Cancer $\% 20$ Staging\%20Manual.pdf.

7. Ellison E, Zollinger RM Jr. Zollinger's Atlas of surgical operations, tenth edition. McGraw Hill Education, 2016:190-229.
8. Farouk R, Nelson H, Radice E, Mercill S, Gunderson L. Accuracy of computed tomography in determining resectability for locally advanced primary or recurrent colorectal cancers. Am J Surg. 1998 Apr;175(4):283-7.

9. Heald RJ, Daniels I. Rectal cancer management: Europe is ahead. Recent Results Cancer Res. 2005;165:75-81.

10. Gunderson LL, Sosin H. Areas of failure found at reoperation (second or symptomatic look) following "curative surgery" for adenocarcinoma of the rectum. Clinicopathologic correlation and implications for adjuvant therapy. Cancer. 1974 Oct;34(4):1278-92.

11. Cass AW, Million RR, Pfaff WW. Patterns of recurrence following surgery alone for adenocarcinoma of the colon and rectum. Cancer. 1976 Jun;37(6):2861-5.

12. Cohen AM, Wood WC, Gunderson LL, Shinnar M. Pathological studies in rectal cancer. Cancer. 1980 Jun 15;45(12):2965-8.

13. Olson RM, Perencevich NP, Malcolm AW, Chaffey JT, Wilson RE. Patterns of recurrence following curative resection of adenocarcinoma of the colon and rectum. Cancer. 1980 Jun 15;45(12):2969-74.

14. Hickey RC, Romsdahl MM, Johnson DE, Wallace S, Mavligit GM, Leavens ME, Borgelt B. Recurrent cancer and metastases. World J Surg. 1982 Sep;6(5):585-95.

15. Pilipshen SJ, Heilweil M, Quan SH, Sternberg SS, Enker WE. Patterns of pelvic recurrence following definitive resections of rectal cancer. Cancer. 1984 Mar 15;53(6):1354-62.

16. Wangensteen $\mathrm{OH}$, Lewis FJ, Arhelger SW, Muller JJ, Maclean LD. An interim report upon the second look procedure for cancer of the stomach, colon, and rectum and for limited intraperitoneal carcinosis. Surg Gynecol Obstet. 1954 Sep;99(3):257-67.

17. Fritsch A, Seidl W, Walzel C, Moser K, Schiessel R. Palliative and adjunctive measures in rectal cancer. World J Surg. 1982 Sep;6(5):569-77. 